\title{
INSERTING TURBO CODE TECHNOLOGY INTO THE DVB SATELLITE BROADCASTING SYSTEM *
}

\author{
Matthew C. Valenti \\ Department of Computer Science and Electrical Engineering \\ West Virginia University \\ Morgantown, WV 26505 \\ mvalenti@wvu.edu
}

\begin{abstract}
This paper illustrates the potential benefits of inserting turbo code technology into emerging communication satellite systems. This paper considers the application of turbo codes to the Digital Video Broadcasting (DVB) standard, which is based on the concatenation of a Reed Solomon code and a convolutional code. We show that by simply replacing the convolutional code with a turbo code, a $1 \mathrm{~dB}$ improvement in coding gain can be achieved in an AWGN channel. This benefit is obtained with no loss in code rate and only a moderate increase in decoder complexity. Furthermore, since Reed-Solomon coding is used to correct burst errors at the output of the turbo decoder, the problematic "bit error rate floor" experienced by unprotected turbo codes is practically eliminated. We then discuss methods for extending the improvement to $2 \mathrm{~dB}$ by using larger frame sizes and more efficient Reed-Solomon codes. We conclude with a brief survey of other methods that can be used to achieve further performance improvements.
\end{abstract}

\section{INTRODUCTION}

Turbo codes, introduced in 1993 by Berrou et al, are an extremely energy efficient error correction technique based on the principles of parallel code concatenation, recursive encoding, nonuniform interleaving, and iterative decoding [1]. Because of their remarkable energy efficiency, turbo codes have generated a considerable amount of attention in recent years. Several scientific and commercial systems have proposed using turbo codes, including the Consultative Committee for Space Data Systems (CCSDS) deep space telemetry recommendation and third generation cellular communication proposals including cdma2000 and UMTS Terrestrial Radio Access (UTRA).

Turbo codes are particularly attractive for military satellite communication systems, where improved energy efficiency

*This research supported by Michael G. Stelling, William E. Snelling, and Eugene E. Estinto of the Coleman Research Corporation (email: Mike_Stelling@mail.crc.com) translates directly into reduced equipment and antenna size requirements as well as extended battery life for portable ground stations. In this paper, we discuss the possibility of inserting turbo code technology into existing military satellite systems. However, in order to keep the discussion at an unclassified level, we have chosen a typical commercial satellite system to use as an example. In particular, the system under consideration is the Digital Video Broadcasting (DVB) system ${ }^{1}$, which was standardized in the early 1990's by the European Broadcasting Union (EBU) and the European Telecommunications Standards Institute (ETSI) [2].

The DVB system is based on the serial concatenation of a shortened Reed Solomon (RS) code and an Odenwalder convolutional code. The benefits of concatenating Reed Solomon and convolutional codes have been well known since Forney's work in the 1960's [3]. Up until the introduction of turbo codes, it was widely believed that the concatenated $\mathrm{RS} /$ convolutional coding approach was the most energy efficient error correction technique that could be implemented with reasonable complexity. Since their introduction, concatenated RS/convolutional codes have been used for deep space communications with the Voyager and Galileo missions to the outer planets, as well as for several satellite communication systems [4]. In this paper, we consider replacing the convolutional codec in the DVB system with a turbo codec. Our simulation results show that a $1 \mathrm{~dB}$ improvement in energy efficiency can be obtained at a bit error rate (BER) of $10^{-7}$ by making such a substitution. Note that although the serial concatenation of RS and turbo codes has been previously discussed in the literature [5], our discussion differs from earlier work in that it targets an actual candidate system (DVB) for the insertion of turbo codes and provides much more specific design recommendations.

One of the known weaknesses of turbo codes is that the BER curve begins to flatten at lower BERs (on the order of $10^{-5}$

\footnotetext{
${ }^{1}$ Since the standardization of the original DVB system, the DVB consortium has introduced several other DVB specifications. The particular variant of DVB that we consider in this paper is DVB-S (for DVB-satellite).
} 


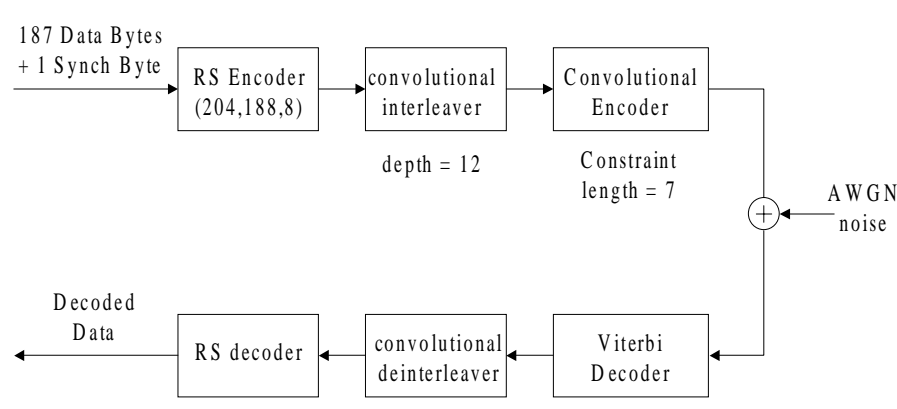

Figure 1: Coding for the DVB standard.

to $\left.10^{-6}\right)$. This behavior is the result of the relatively small minimum distance of turbo codes [6]. However, because we have maintained the Reed Solomon outer code in the modified DVB system, the BER floor is lowered well below our target BER of $10^{-7}$. Thus the Reed Solomon code plays an important role in the new system, and its contribution to performance should not be neglected. However, the Reed Solomon code proposed in the DVB standard is far from optimal when used with turbo codes. Thus, we propose other Reed Solomon codes which offer improved performance over the original DVB code. In particular, we consider full length RS codes (rather than shortened ones) and different values of $t$ (the error correction capability of the RS code).

Finally, it should be noted that the performance of a turbo code is greatly influenced by the size of its interleaver, or equivalently the size of the data frame. While the size of the data frame of the original turbo code was about 64 kilobits, the size of the DVB data frame is less than 2 kilobits. Additional improvements can be obtained by using larger frame sizes, which can be achieved by allowing the input of the turbo encoder to be composed of multiple Reed Solomon code words. In particular, we found that a gain of approximately $2 \mathrm{~dB}$ over the DVB standard (at BER $=10^{-7}$ ) is possible by using a 64 kilobit turbo code frame size.

In the first part of this paper, we give an overview of the DVB standard and introduce the concept of turbo coding. Next, we discuss the straightforward replacement of the DVB convolutional codec with a turbo codec. This is followed by a discussion of methods for extending the coding gain by reconsidering the choice of Reed Solomon code and using larger turbo code frames. Finally we conclude by highlighting additional strategies that can be used to further improve performance.

\section{THE DVB STANDARD}

As shown in Fig. 1, the DVB encoder consists of an outer Reed Solomon encoder and an inner convolutional encoder that are separated by a convolutional interleaver [2]. Data from the source (assumed to be a randomized MPEG-2 stream $^{2}$ ) is first segmented into blocks of 187 bytes. A synch byte is attached, and the resulting message is encoded by a shortened $(204,188)$ Reed Solomon code, which is capable of correcting $t=8$ byte errors. The 204 byte Reed Solomon code word is passed through a convolutional interleaver of depth 12. Next, the output of the interleaver is encoded by an Odenwalder convolutional encoder, which has constraint length $K=7$ and octal generators $(171,133)$. The inner (convolutional) encoder can operate at rates $r=1 / 2,2 / 3$, $3 / 4,5 / 6$, and $7 / 8$, with the higher rate codes obtained by puncturing the mother $r=1 / 2$ code. For the remainder of this paper, we will focus our attention on the rate $r=1 / 2$ inner encoder, although we plan to consider the $r=7 / 8$ code in future work.

The modulation used by DVB is gray-coded QPSK with square-root raised cosine rolloff pulse shaping (rolloff factor $\alpha=0.35$ ). For the simulation results presented in subsequent sections, we assume QPSK modulation with perfect carrier/phase synchronization, symbol timing, and frame synchronization. Under these assumptions and the assumption of perfect pulse shaping filters at the transmitter and receiver, the effects of pulse shaping can be neglected. To provide a fair comparison, these ideal conditions are assumed for our simulations of both the original DVB standard as well as our modified versions of it. In the DVB specification, a 0.8 $\mathrm{dB}$ modem implementation margin is included to account for suboptimal synchronization and filtering effects. We do not consider such implementation loss in this study, but intend to consider it in our future work.

The DVB decoder is also shown in Fig. 1 and is just the reverse of the encoder. While the DVB specification does not make specific design recommendations regarding the decoder, the convolutional code is decoded using the Viterbi algorithm while the Reed Solomon code is (usually) decoded using the Berlekamp-Massey algorithm.

\section{TURBO CODES}

A typical turbo encoder is shown in Fig. 2. The turbo encoder consists of two recursive systematic convolutional (RSC) encoders that are fed the same set of data in parallel, although in a different order. An RSC encoder may be derived from a conventional (nonrecursive) convolutional encoder by feeding back one of its outputs. The two RSC encoders are usually, although not necessarily, the same. The interleaver separating the two encoders changes the ordering of the lower encoder's input according to a prescribed nonuniform permutation function. To distinguish this interleaver from the interleaver separating the inner and outer code in Fig. 1, we will refer to it as the "turbo interleaver". The size of the turbo interleaver matches the size of the data

\footnotetext{
${ }^{2}$ In DVB, the MPEG-2 source is randomized with a pseudorandom binary sequence in order to ensure sufficient phase transitions in the QPSK modulation.
} 


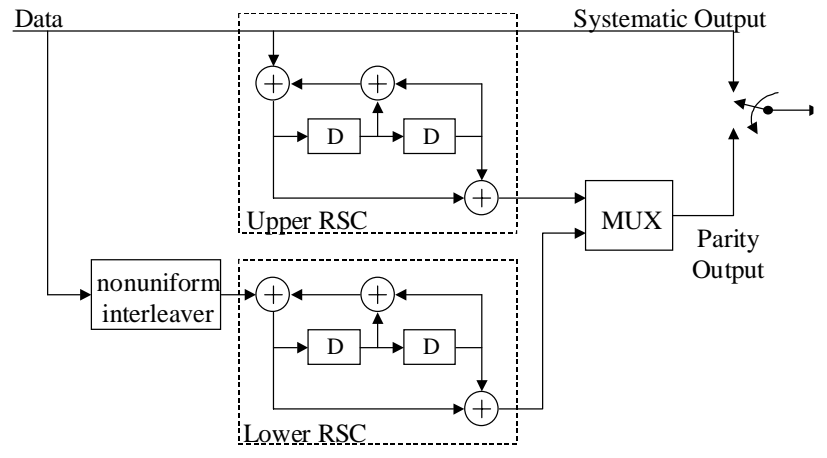

Figure 2: Turbo Encoder.

frame (with the possible inclusion of tail bits) at the input to the turbo encoder. As we will show in Section V, the performance of a turbo code improves with increasing frame size, or equivalently increasing turbo interleaver size. Because the systematic outputs of the two encoders are identical (only in a different order), only the upper encoder's systematic output must be transmitted. The resulting code is rate $1 / 3$, although it can be increased to rate $1 / 2$ by multiplexing the outputs (i.e. only transmitting the odd-indexed parity bits of the upper encoder and the even-indexed parity bits of the lower encoder).

As with conventional convolutional codes, it is desirable to bring the encoder back to the all-zeros state after encoding each data frame. This can be done by appending a small number of tail bits at the end of each frame. However, unlike conventional convolutional codes, the tail bits for a turbo code are not necessarily all zeros. Because of the recursive nature of the encoders and the nonuniform interleaving, it is difficult to bring both encoders back to the all-zeros state. Thus, we chose to only terminate the upper encoder with $K-1$ tail bits ( $\mathrm{K}$ is the constraint length), while leaving the lower encoder's trellis open.

The decoder (not shown) uses the concept of iterative decoding. Two elementary soft-input soft-output (SISO) decoding modules are present in the receiver, one for each RSC encoder in the transmitter. Decoding begins with the first SISO module deriving a posteriori probability (APP) estimates based on its received symbols. These estimates, typically in the form of a log-likelihood ratio (LLR), are then passed to the second decoder to be used as a priori information. The second decoder then uses this side information in conjunction with its received symbols to produce its own LLR of the data. The LLRs from the second decoder are passed back to the first decoder to be used as side information during the next iteration. Decoding proceeds in an iterative fashion for a fixed number of conditions or until a halting condition is reached (such as zero bit errors in the frame). While a variety of algorithms can be used to implement the SISO modules, including SOVA and MAP, we have chosen to implement to modules using the log-MAP algorithm [7].

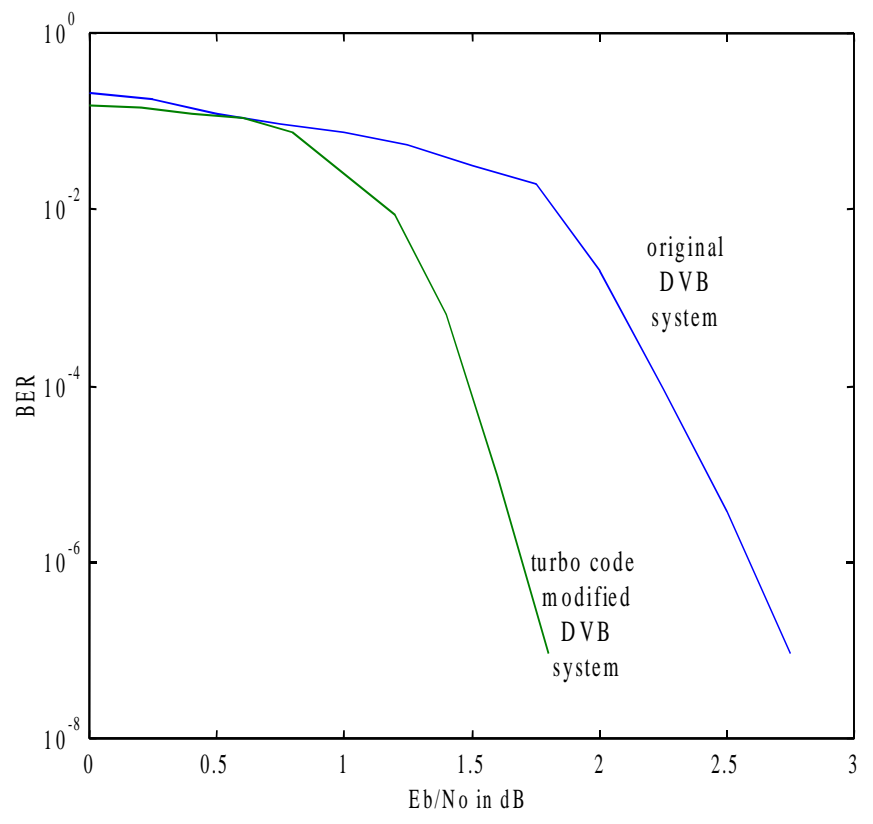

Figure 3: Bit error performance using convolutional inner code (original DVB system) and turbo inner code (modified DVB system). Both systems use a $(204,188,8)$ outer $\mathrm{RS}$ code, a rate $1 / 2$ inner code, and a depth 12 convolutional interleaver between inner and outer code.

\section{TURBO CODE ENHANCED DVB}

Our first modification to the DVB standard was to simply replace the convolutional encoder in Fig. 1 with a turbo encoder. As in [1], the turbo code uses constraint length $K=$ 5 constituent RSC encoders with octal feedback generator (37) and feedforward generator (21). The size of the turbo interleaver is 1636 bits, the first $8 \times 204=1632$ corresponding to the output of the RS encoder and the remaining 4 bits corresponding to a tail used to terminate the trellis of the upper encoder. The interleaver is an "S-random" or "spread" interleaver with $S=21$ [8]. At the receiver, 12 iterations of $\log$-MAP decoding are performed [7].

Simulation results are shown in Fig. 3. Here the performance of the concatenated RS/turbo code is compared with the performance of the RS/convolutional code used by DVB. In particular, the bit error rate (BER) is plotted against the ratio of energy per data bit $\left(E_{b}\right)$ to single-sided noise spectral density $\left(N_{o}\right)$. As can be seen, by substituting a turbo code for the convolutional code, a $1 \mathrm{~dB}$ increase in coding gain at BER $=10^{-7}$ is achieved. The only system modification required to achieve this gain is for the inner code to be changed; the remainder of the system remains intact.

Note that this gain comes at the cost of a modest increase in decoder complexity. While the complexity to decode the $K=7$ convolutional code is $O\left(2^{7}\right)$, the complexity to decode the $K=5$ turbo code is $O\left(4 \times 2^{5}\right)$ per iteration. Thus for 12 
full iterations, the complexity of the turbo code is 12 times that of the convolutional code. However, in practice, it might not be necessary to perform 12 decoder iterations for every frame. Instead, each frame could be iteratively decoded until either its BER drops to zero or the maximum number of iterations is reached. Several methods could be used to measure the BER, including the use of a short CRC error detecting code or using a cross-entropy measure [9]. Such a strategy results in a system whose computational load varies from frame to frame, but is never more than 12 times that of the original convolutional code. In general, more iterations are required at low signal to noise ratio (SNR) and less are required at higher SNR.

\section{IMPROVED TURBO CODE BASED DVB}

While the $(204,188,8)$ shortened Reed Solomon code provides adequate performance when coupled with a convolutional code, it is not necessarily the best outer code to use in conjunction with a turbo code. In particular, use of this outer code results in a $0.35 \mathrm{~dB}$ penalty in energy efficiency due to the fractional rate $\operatorname{loss}$ (i.e. $10 \log (204 / 188)$ ). This penalty can be reduced to $0.28 \mathrm{~dB}$ without sacrificing error correction capability by using a full length $(255,239,8)$ Reed Solomon code. While the performance of concatenated $\mathrm{RS}$ /convolutional codes generally improves with increasing $\mathrm{t}$ (the error correcting capability of the RS code), this is not necessarily true for concatenated RS/turbo codes. Thus the penalty due to the outer code rate loss can be reduced further by using a less powerful $(255,255-2 \mathrm{t}, \mathrm{t}) \mathrm{RS}$ code, where $t<8$.

In the second part of this study, we considered using a full length $(255,255-2 \mathrm{t}, \mathrm{t}) \mathrm{RS}$ code in conjunction with a turbo code using a 2044 bit spread interleaver $(8 \times 255$ data bits +4 tail bits). Again, a convolutional encoder of depth 12 is placed between the inner and outer decoder, and the turbo decoder uses 12 iterations of the log-MAP algorithm. Simulation results are shown in Fig. 4 for various values of $t$ as well as for a turbo code without an outer RS code. It is interesting to note that in the "waterfall" region of the curve, performance is actually worse for increasing t due to the lower rate of the outer code. However, the unprotected turbo code encounters a BER "floor" at about $10^{-5}$ or $10^{-6}$. The benefit of using the outer RS code is that it lowers this floor with increasing t. From this curve we can conclude that by only using a $(255,247,4)$ outer RS code, a BER of $10^{-7}$ can be achieved for $E_{b} / N_{o} \approx 1.55 \mathrm{~dB}$, which is about $1.2 \mathrm{~dB}$ better than the DVB standard.

Next, it should be noted that the performance of a turbo code improves with increasing frame (turbo interleaver) size. Thus, in order to obtain more than a $1.2 \mathrm{~dB}$ increase in coding gain over the DVB standard, larger turbo codes must be considered. In the final stage of this study, we considered turbo codes whose frame sizes are integer multiples of the RS

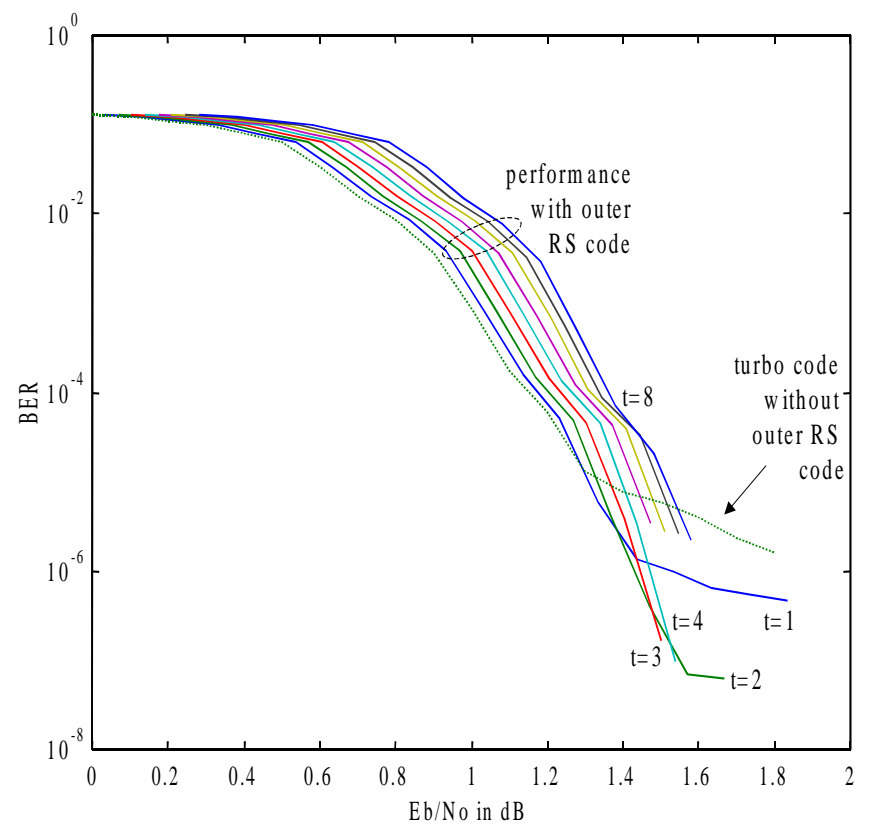

Figure 4: Performance of serial concatenation of $(255,255-2 \mathrm{t}, \mathrm{t})$ RS code and length 2044 turbo code. A depth 12 convolutional interleaver separates inner and outer codes. Performance is shown for $1 \leq t \leq 8$ as well as for an unprotected turbo code.

code word size. In particular, we consider a 16,324 bit turbo code $(8 \times 2040+4)$ and a 65,284 bit turbo code $(32 \times 2040+$ 4). In both cases a spread turbo interleaver is used, and 12 iterations of log-MAP decoding performed at the receiver. In this case, however, the convolutional interleaver between inner and outer encoder is removed. We found that this interleaver did not improve performance because it tended to only interleave symbols within the same turbo code word.

Simulation results are shown for the 16,234 bit turbo code in Fig. 5 and for the 65,284 bit turbo code in Fig. 6. For the 16,234 bit turbo code, we find that a $(255,247,4)$ outer RS code can achieve a BER of $10^{-7}$ at $E_{b} / N_{o} \approx 0.95 \mathrm{~dB}$, or about $1.8 \mathrm{~dB}$ better than the DVB standard. For the 65,284 bit turbo code, only a $(255,251,2)$ outer RS code is necessary to meet the target $\mathrm{BER}$ at $E_{b} / N_{o} \approx 0.75 \mathrm{~dB}$, which is a 2 $\mathrm{dB}$ gain over DVB.

\section{CONCLUSIONS}

By using a turbo code in conjunction with an outer Reed Solomon code, energy efficiencies can be achieved that are 2 $\mathrm{dB}$ superior to the DVB standard. For modest target BERs, such as $10^{-5}$, use of an outer RS code may actually hurt performance, but at lower BERs it has the benefit of lowering the error floor. Because the turbo decoder is capable of producing bit reliability information, it should be possible to improve performance further by using errors-and-erasures decoding of the RS code. 


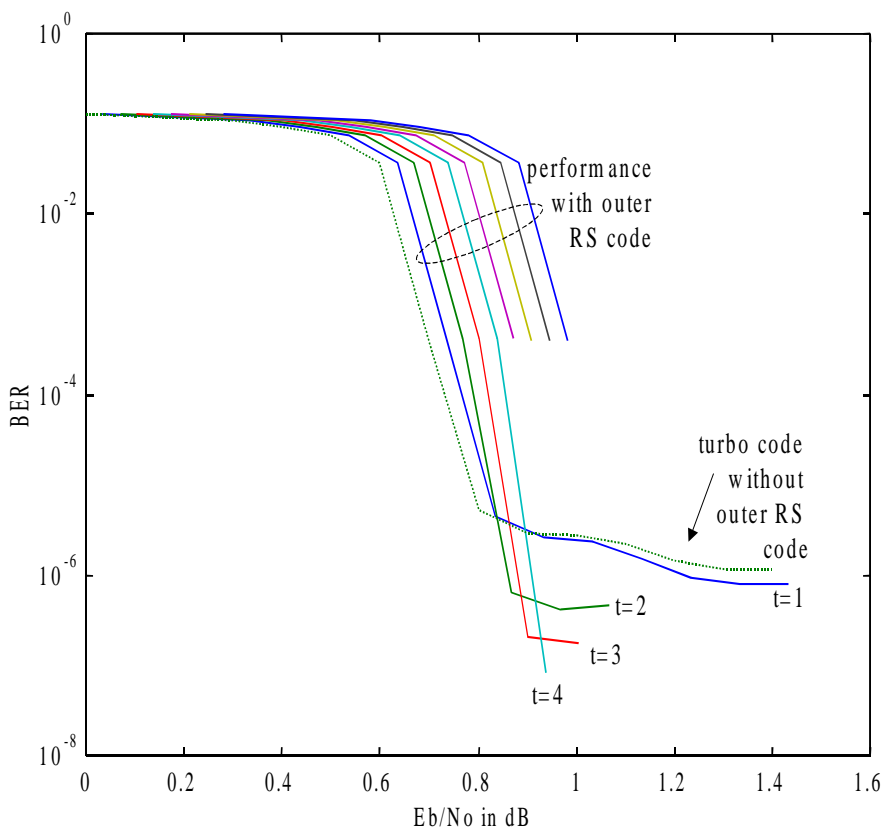

Figure 5: Performance of serial concatenation of $(255,255-2 \mathrm{t}, \mathrm{t})$ RS code and length 16,234 turbo code.

It should be noted that there are other methods for lowering the BER floor of a turbo code aside from using an outer RS code. For instance, one could use serially concatenated convolutional codes (SCCC), also called serial turbo codes [10]. However, the waterfall region of SCCC codes tends to be at a higher $E_{b} / N_{o}$ than conventional parallel concatenated turbo codes and it is doubtful that such an approach will be able to yield a $2 \mathrm{~dB}$ improvement over DVB. Another option is to protect the turbo code with an outer $\mathrm{BCH}$ code, rather than a RS code [11]. The logic is that, while the Viterbi algorithm tends to produce bursts of errors that are well matched to RS codes, the MAP algorithm used for turbo decoding produces more randomly distributed errors that may be better matched to $\mathrm{BCH}$ codes. An interesting twist on this idea is that due to the unequal error protection property of turbo codes, some of the bits at the output of the turbo decoder will almost never be in error. Thus a shorter BCH code can be used to only protect those bits that are most likely to be in error [9].

\section{REFERENCES}

[1] C. Berrou, A. Glavieux, and P. Thitimasjshima, "Near Shannon limit error-correcting coding and decoding: Turbo-codes(1)," in Proc., IEEE Int. Conf. on Commun., (Geneva, Switzerland), pp. 1064-1070, May 1993.

[2] European Telecommunications Standards Institute, "Digital broadcasting system for television sound and data services," ETS 200 421, Dec. 1994.

[3] G. D. Forney, Concatenated Codes. Cambridge, MA: MIT Press, 1966.

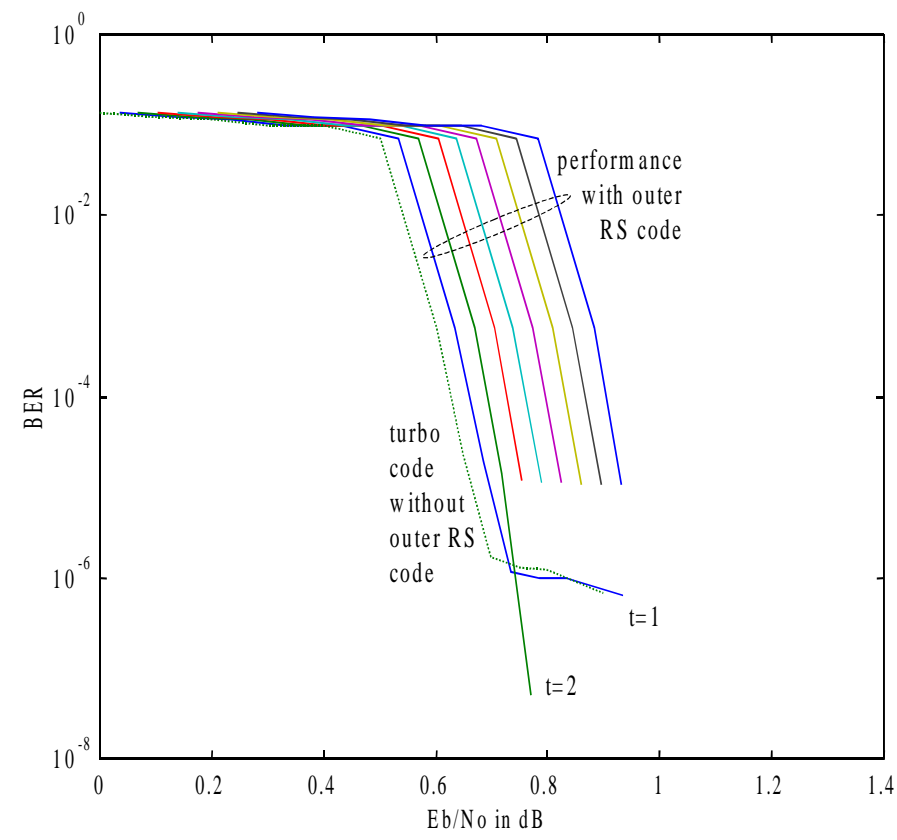

Figure 6: Performance of serial concatenation of $(255,255-2 \mathrm{t}, \mathrm{t})$ RS code and length 65,284 turbo code.

[4] D. J. Costello, J. Hagenauer, H. Imai, and S. B. Wicker, "Applications of error-control coding," IEEE Trans. Inform. Theory, vol. 44, pp. 2531-2560, Oct. 1998.

[5] D. J. Costello and G. Meyerhans, "Concatenated turbo codes," in Proc., IEEE Int. Symp. on Inform. Theory and Appl., (Victoria, Canada), pp. 571-574, Sept. 1996.

[6] L. C. Perez, J. Seghers, and D. J. Costello, "A distance spectrum interpretation of turbo codes," IEEE Trans. Inform. Theory, vol. 42, pp. 1698-1708, Nov. 1996.

[7] P. Robertson, P. Hoeher, and E. Villebrun, "Optimal and sub-optimal maximum a posteriori algorithms suitable for turbo decoding," European Trans. on Telecommun., vol. 8, pp. 119-125, Mar./Apr. 1997.

[8] S. Dolinar and D. Divsalar, "Weight distributions for turbo codes using random and nonrandom permutations," JPL TDA Progress Report, vol. 42, pp. 56-65, Aug. 15th 1995.

[9] K. R. Narayanan and G. L. Stuber, "Selective serial concatenation of turbo codes," IEEE Commun. Letters, vol. 1, pp. 136-140, Sept. 1997.

[10] S. Benedetto and G. Montorsi, "Serial concatenation of block and convolutional codes," Electronics Letters, vol. 32, pp. 887-888, May 9th 1996.

[11] J. D. Andersen, "Turbo codes extended with outer BCH code," Electronics Letters, vol. 32, pp. 2059-2060, Oct. 24th 1996. 